\title{
Turning the attentional blink on and off: Opposing effects of spatial and temporal noise
}

\author{
Elwyn W. Martin • James T. Enns • Kimron L. Shapiro
}

Published online: 19 February 2011

(C) Psychonomic Society, Inc. 2011

\begin{abstract}
Brain oscillations in various frequency bands have been shown to be an important means of enabling interarea communication for high-level cognitive performance. Interestingly, perturbation to such oscillations in the form of weak noise has been shown to benefit perception in tasks such as the attentional blink (AB). Here, we investigated perturbation intrinsic to the $\mathrm{AB}$ task in two conditions in which noise arose from either temporal or spatial discontinuity. Consistent with theoretical predictions, temporal discontinuity resulted in a reduced $\mathrm{AB}$, whereas spatial discontinuity resulted in an increased $A B$. The results are discussed in the framework of both stochastic resonance theory and pretarget alpha oscillations, two neurally based accounts of perceptual processing.
\end{abstract}

Keywords Attentional Blink (AB) · Spatial discontinuity · Temporal discontinuity $\cdot$ Stochastic resonance $\cdot$ Alpha oscillations · Noise

\section{Introduction}

The attentional blink (AB; Raymond, Shapiro, \& Arnell, 1992) refers to a reduction in the accuracy of reporting the

E. W. Martin · K. L. Shapiro

Bangor University Centre for Cognitive Neuroscience,

Wales, UK

J. T. Enns

Department of Psychology, University of British Columbia,

Vancouver, BC, Canada

K. L. Shapiro $(\square)$

School of Psychology, Bangor University,

Penrallt Road, Brigantia Building,

UK, Wales LL57 2AS

e-mail: k.shapiro@bangor.ac.uk second of two targets $(\mathrm{T} 1, \mathrm{~T} 2)$ when they are presented by rapid serial visual presentation (RSVP). The reduction is maximal when the targets are separated in time by approximately $100-500 \mathrm{~ms}$, although accuracy for $\mathrm{T} 2$ can be equivalent to that for $\mathrm{T} 1$ when participants are instructed to ignore $\mathrm{T} 1$, when nontarget items immediately following the targets are omitted, and/or when the temporal separation between the targets exceeds $\sim 500 \mathrm{~ms}$ (cf. Raymond et al., 1992; Seiffert \& Di Lollo, 1997). The present study explored two experimental factors that have diametrically opposite effects on the $\mathrm{AB}$ : one reducing the $\mathrm{AB}$ to levels usually observed only when participants are instructed to ignore $\mathrm{T} 1$, and the other increasing the $\mathrm{AB}$ by an equivalent amount.

A wide variety of accounts have been advanced to explain the AB (cf. Dux \& Marois, 2009), but the point of departure for the present study was inspired by a growing body of evidence regarding the role of neural oscillations in perception (e.g., Busch, Dubois, \& VanRullen, 2009; Buzsáki, 2004). For example, stochastic resonance theory (Gammaitoni, Hanggi, Jung, \& Marchesoni, 1998; Wiesenfeld \& Moss, 1995) is a putative mechanism whereby neural noise arising from external sources, normally a negative factor, can, under the right circumstances, increase signal detection. Recent behavioral studies have reported that detection of a weak visual target is enhanced by the presence of randomly flickering gray-level noise adjacent to two possible target locations (e.g., Kitajo, Yamanaka, Ward, \& Yamamoto, 2006).

Although the application of stochastic resonance to behavioral outcomes in cognitive psychology is in its infancy, a recent study of the $\mathrm{AB}$ suggests that doing so can be profitable. Kawahara (2009) found that "noise" in the form of nontarget distractors surrounding the rapid serial visual presentation (RSVP) stream and continuously present could both increase and decrease $\mathrm{AB}$ magnitude. When the noise arose from a dimension different from that 
of the target detection requirement, $\mathrm{T} 2$ detection was enhanced. In contrast, when the noise arose from the same dimension as targets, a larger $\mathrm{AB}$ resulted. Stochastic resonance received support as a viable account, in that the type of distractor influenced the direction of the effect and the effect occurred only for targets in the temporal window defining the $\mathrm{AB}$ (see the Discussion section for a further treatment of these issues).

The noise in the experiments described above arose from sources in the periphery of the RSVP stream. ${ }^{1}$ However, noise generated by experimental stimuli themselves may also exert an influence but have not yet been investigated in the context of the $\mathrm{AB}$. Importantly, precise experimental control over such noise allows one to determine whether the attentional modulation observed in the $\mathrm{AB}$ is related directly to events preceding each of the targets, or whether the modulation is a consequence of a more continuous state, as described above. To accomplish this goal, intrinsic distraction was presented in the RSVP stream either (1) prior to T1, (2) between T1 and $\mathrm{T} 2$, or (3) in both parts of the stream. A comparison of the second with the first and third of these conditions enabled us to examine whether the dramatically different outcomes of such intrinsic noise arise solely from effects on $\mathrm{T} 1$, which, by some accounts of the $\mathrm{AB}$, can predict $\mathrm{T} 2$ performance or from effects on both targets. The goal of the present report is to gain a better understanding of the role of noise in high-level perception by carefully controlling the source in the specific context of the $\mathrm{AB}$ paradigm.

For our beneficial noise, we chose to manipulate the temporal rhythm of the RSVP stream. As has been suggested by Kawahara (2009), a dimension irrelevant to the target task should enhance perception. For our detrimental noise, again following Kawahara, we chose to introduce a degree of variability similar to that induced by manipulating the temporal rhythm, but from a dimension directly relevant to the performance of the imperative task (i.e., identifying a spatially defined letter target in the RSVP stream). Variability in item size should increase the amount of attention that participants devote to $\mathrm{T} 1$, because size variation makes shape identification less efficient and more effortful (Schultz \& Eriksen, 1978).

\section{Method}

Participants

Twenty-one undergraduate psychology students (15 females; mean age, 19.7 years; normal or corrected-to-

\footnotetext{
${ }^{1}$ Kawahara's (2009) manipulation arose from stimuli spatially proximal to the $\mathrm{AB}$ task but not from the actual stimuli involved in the task per se.
}

normal visual acuity) from Bangor University volunteered to participate.

\section{Apparatus}

Stimuli were presented on a $1,024 \times 768$ pixel, 32-bit color quality, 17-in. cathode ray tube (CRT) monitor using EPrime version 1.1 experimental software (Psychology Software Tools, Pittsburgh, PA). All the stimuli were presented in intervals of the $17-\mathrm{ms}$ refresh rate of the monitor.

Task

A stream of 24 or $25^{2}$ letters was presented in the center of a gray screen, and participants attempted to identify two targets. All items in the stream were black, with the exception of the two white targets (T1 and T2). Nontargets were drawn from a randomly alternating sequence of the entire alphabet, with the exceptions of $\mathrm{B}, \mathrm{G}, \mathrm{S}, \mathrm{X}, \mathrm{K}$, and $\mathrm{Y}$. The letters B, G, and $\mathrm{S}$ were used as randomly selected $\mathrm{T} 1$ items; $\mathrm{X}, \mathrm{K}$, and $\mathrm{Y}$ were used as randomly selected $\mathrm{T} 2$ items. T1 always appeared as the 12th item in the stream in order to equate the amount of discontinuity prior to $\mathrm{T} 1$.

Noise in the form of discontinuity was added to the temporal and spatial domain of the stream as described below and shown in Fig. 1a. The location of the temporal noise was systematically varied in three blocked conditions: across all pre- $\mathrm{T} 1$ items only, between the $\mathrm{T} 1+1$ item and $\mathrm{T} 2$, and in both pre-T1 and pre-T2 items. A standard (canonical $\mathrm{AB}$ ) condition with no noise served as the baseline, yielding seven conditions (2 [spatial, temporal] $\times$ 3 [pre-T1, pre-T2, both pre-T1 and pre-T2], plus baseline). Each participant completed all seven conditions in one of seven Latin square counterbalanced orders to balance carryover effects. In all conditions, 20 trials were completed for each of three possible (randomly presented) lag positions of T2: lag 2 (i.e., $204 \mathrm{~ms}$ elapsed between the onset of T1 and T2), lag 3 (306 ms), and lag 7 (714 ms). Each participant completed 420 trials.

Temporal noise

Temporal noise was introduced by varying the interstimulus-interval (ISI) between specific nontarget items as described above and shown in Fig. 1a, leaving a constant display duration $(17 \mathrm{~ms})$ for every item. Here, the letter font size was identical to that in the standard condition (20-point

\footnotetext{
${ }^{2}$ Temporal noise lag 2 trials required two items during the intertarget interval to manipulate temporal noise while holding the T1-mask ISI constant. This could not be implemented in the spatial condition, since it would interrupt the required temporal regularity.
} 
Fig. 1 Schematic illustration of the seven conditions in the experiment, with the standard condition shown twice $(\mathrm{T} 1=$ first target; $\mathrm{T} 2=$ second target). The standard condition had no variability in either the temporal or the spatial domain and was similar to canonical $\mathrm{AB}$ stimulus conditions. Noise was introduced in one of two ways (a temporal discontinuity, b spatial discontinuity) with three variations (before T1, before T2 [i.e., after the T1 mask and before T2], and before both targets [i.e., before T1 and before T2]). Temporal noise is illustrated for only a part of the stimulus stream by the irregular interval between nontarget items. Spatial noise is illustrated by the height of the bars representing font size. Note that T1, T2, and their respective masks were identical with respect to all stimulus items (targets and nontargets) in the standard condition a Temporal Discontinuity

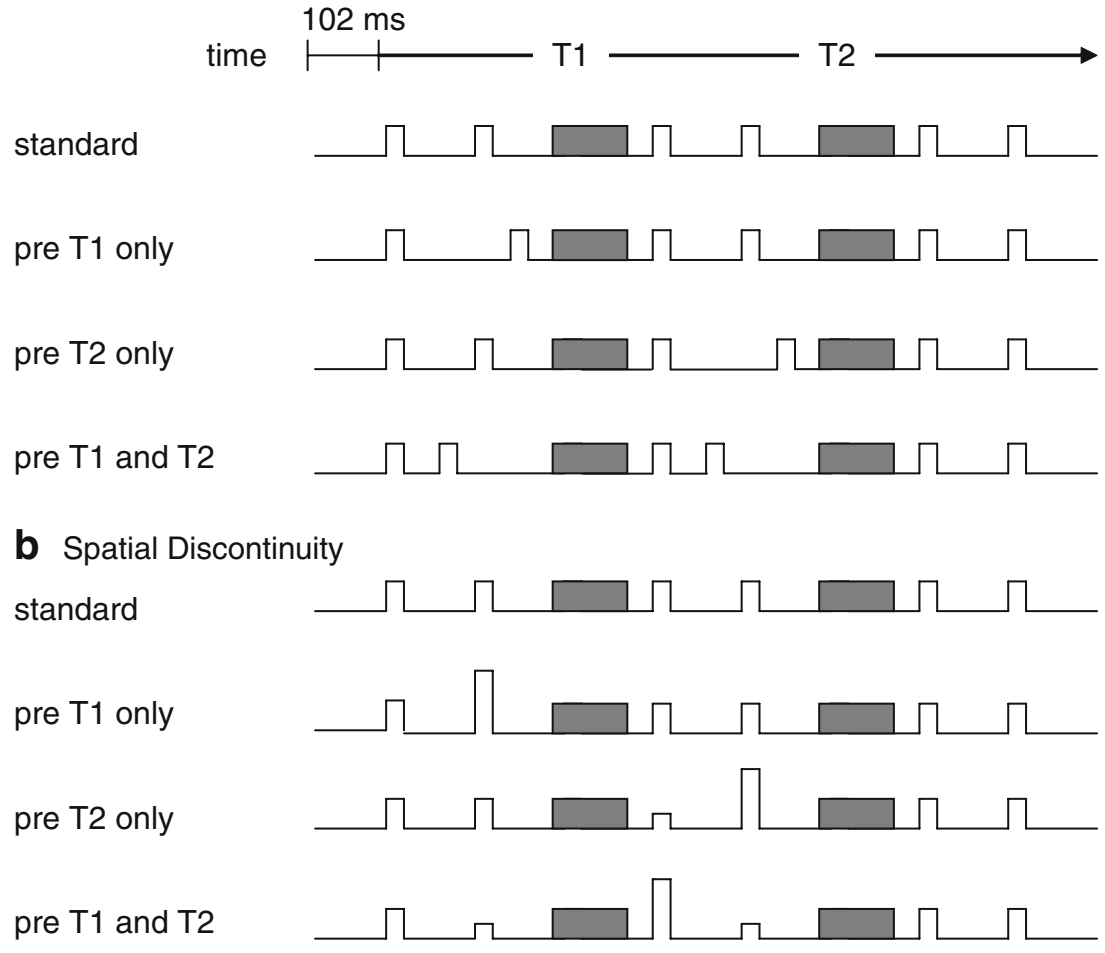

font), as was the display duration. The mean stimulus onset asynchrony (SOA) was identical to that in the standard condition (102 ms; composed of a mean ISI of $85 \mathrm{~ms}$ plus the 17-ms display duration). Temporal noise was created in each stream by selecting randomly from a predetermined uniform distribution of ISIs (17-51-85-119-153 ms). The two exceptions to the random selection of ISIs in the temporal noise conditions were (1) the ISI between each target and its respective mask, to maintain target/mask equality across all conditions, and (2) the ISI occurring between items following T2's mask; these were presented with an 85-ms ISI, under the assumption that these items have no influence on target performance.

Spatial noise

Spatial noise was introduced by varying the font size of specific nontarget letters as described below and as illustrated in Fig. 1b. Here, the average font size was nearly identical to that used in the standard condition (i.e., a 20point font), with the varying font sizes selected randomly in each stream from $16,18,20$, or 22 points. Two exceptions to the random selection of font size in the spatial noise condition were (1) T1, T2, and their respective masks, since these were kept the same as those in the canonical condition to maintain equality across targets and masks in all conditions, and (2) the items occurring subsequent to the $\mathrm{T} 2+1$ item, since these were all in 20-point font, under the assumption that items following the T2 mask have no influence on target performance. In all three spatial noise conditions, as in the standard, all stimuli were presented at $\sim 10$ items per second $(\mathrm{SOA}=102 \mathrm{~ms})$.

\section{Results}

T1 and T2 accuracy were examined separately for each of the temporal and spatial noise conditions with a mixed-design ANOVA involving four conditions (baseline, pre-T1, pre-T2, pre-T1 + pre-T2) $\times 3$ lags $(204,306,714 \mathrm{~ms}) \times 7$ orders. All statistical tests were deemed significant at the .05 level, and all post hoc tests used a Bonferroni correction.

Temporal noise

T1 accuracy Temporal noise generally led to increases in T1 accuracy, as reflected by a significant main effect of condition $F(3,42)=17.45, M S E=45.33, p<.001$. There was no effect of lag, $F(2,28)=2.77, M S E=50.14, p=.08$, and no interaction between condition and lag, $F(6,84)=.53$, $M S E=61.06, p=.78$, nor was there any effect of order (of conditions) $F(6,14)=0.45, M S E=65.17, p=.82$, or an interaction between order and condition, $F(18,42)=.90$, $M S E=45.33, p=.57$, or order and lag, $F(12,28)=.95$, $M S E=50.14, p=.50$. Post hoc comparisons revealed that relative to the standard condition, T1 accuracy benefited from pre-T1 noise (see Fig. 2a), but not from noise that occurred only pre-T2. 
a

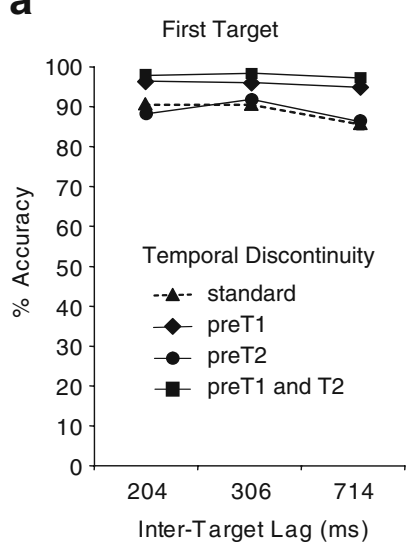

b

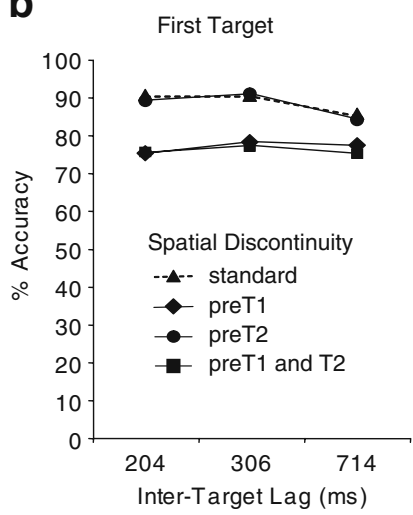

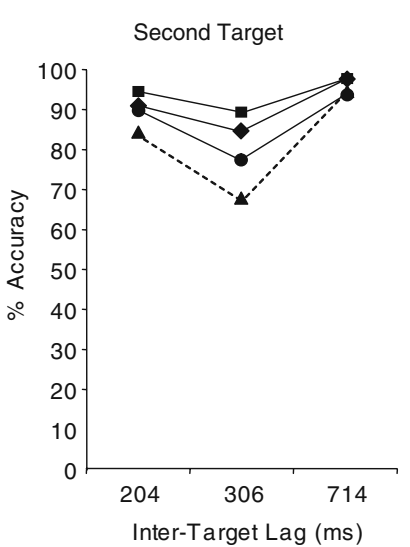

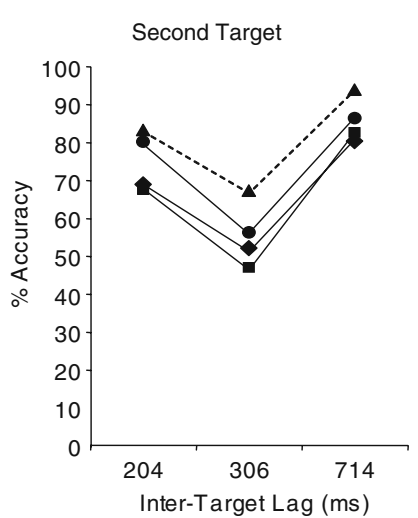

Fig. 2 Mean accuracy for each target (first, second) as a function of lag $(204,306,714 \mathrm{~ms})$ for a temporal noise and b spatial noise. Standard deviations are given in the detailed tables of results (Table 1)

T2 accuracy Temporal noise also led to increases in T2 accuracy, as reflected by significant main effects of condition $F(3,42)=43.45, M S E=36.33, p<.001$, and lag, $F(2,28)=$ 122.53, $M S E=44.99, p<.001$, and an interaction between condition and lag $F(6,84)=10.69, M S E=34.19, p<.001$. There was no effect of order, $F(6,14)=1.22, M S E=38.65$, $p=.35$, and no interaction of condition and order, $F(18,42)=$ $0.97, M S E=36.33, p=.50$, or of lag and order $F(12,28)=$ $0.30, M S E=44.99, p=.98$. Post hoc comparisons revealed that relative to the standard condition, T2 accuracy was significantly improved by temporal noise at lag 3 in all nonbaseline conditions (see Fig. 2a).

T1 and T2 correlations Table 1 shows the correlations between $\mathrm{T} 1$ and $\mathrm{T} 2$ accuracy at all lags. The only statistically significant correlation was positive at lag 7 for pre-T1 noise trials.

Spatial noise

T1 accuracy Spatial noise generally led to decreases in T1 accuracy, as reflected by a significant main effect of

condition, $F(3,42)=62.80, M S E=53.24, p<.001$. There was no effect of lag, $F(2,28)=2.42, M S E=63.39, p=$ .10 , nor was an interaction between condition and lag, $F(6$, $84)=1.05, M S E=59.29, p=.39$, an effect of order, $F(6$, $14)=2.69, M S E=35.11, p=.05$, or an interaction of order and condition, $F(18,42)=1.56, M S E=53.24, p=$ .15 , or order and lag, $F(12,28)=1.02, M S E=63.39, p=$ .52 , found. Post hoc comparisons revealed that, relative to the standard condition, T1 accuracy was reduced by preT1 noise (see Fig. 2b) but not by noise that occurred between $\mathrm{T} 1$ and $\mathrm{T} 2$.

T2 accuracy Spatial noise also led to decreases in T2 accuracy, as reflected by significant main effects of condition, $F(3,42)=71.30, M S E=45.29, p<.001$, and lag, $F(2,28)=387.92, M S E=52.44, p<.001$, and by a significant interaction between lag and condition, $F(6,84)=$ 4.06, MSE $=32.52, p<.001$. There was no effect of order $F$ $(6,14)=0.519, M S E=24.95, p=.78$, and no interaction of condition and order, $F(18,42)=0.76, M S E=45.29, p=.72$, or of lag and order, $F(12,28)=0.71, M S E=52.44, p=.73$. Post hoc comparisons revealed that relative to the standard condition, T2 accuracy was significantly decreased at lag 3 in all but the pre-T2 condition involving spatial noise (see Fig. 2b).

T1 and T2 correlations Table 1 shows the correlations between $\mathrm{T} 1$ and $\mathrm{T} 2$ accuracy at all lags. There were significant positive correlations at lags 2 and 3 in the pre$\mathrm{T} 1$ condition, indicating $\mathrm{T} 1$ and $\mathrm{T} 2$ accuracy rising and falling together. Moreover, there was a significant negative correlation at lag 3 when the spatial noise occurred pre-T2.

\section{Discussion}

The present study demonstrates that noise in a rapid visual stream of stimuli can have dramatically opposite effects during an $\mathrm{AB}$ task, depending on the type of intrinsic discontinuity. As is shown in Fig. 2a, temporal noise generally increased T2 accuracy, with the benefit arising when the noise occurred prior to $\mathrm{T} 1$ and when it occurred prior to the T2 and with a combined benefit when it occurred in both stream locations.

The opposite consequence was observed when the noise arose from spatial, rather than temporal, discontinuity. Variation in the size of nontarget letters in the stream produced a decrease in T2 accuracy, one that was comparable in magnitude to the increase caused by temporal noise. Fig. 2b shows that larger impairments were observed when the noise occurred prior to T1 than when it occurred prior to $\mathrm{T} 2$ and that, in a mirror reflection of the 
Table 1 Correlations between first- and second-target performance

Two-tailed Pearson correlations between first- and second-target (T1 and T2) performance. The designation "*" indicates a significant correlation relationship

\begin{tabular}{|c|c|c|c|}
\hline \multirow{2}{*}{$\frac{\text { Condition }}{\text { Temporal discontinuity Pre-T2 only }}$} & \multicolumn{3}{|c|}{ Pearson correlation two-tailed } \\
\hline & $\begin{array}{l}\text { Lag } 2 \\
r=.39, p=.80\end{array}$ & $\begin{array}{l}\text { Lag } 3 \\
r=-.082, p=.72\end{array}$ & $\begin{array}{l}\text { Lag } 7 \\
r=.27, p=.23\end{array}$ \\
\hline Temporal discontinuity Pre-T1 only & $\begin{array}{l}\text { Lag } 2 \\
r=-.03, p=.90\end{array}$ & $\begin{array}{l}\text { Lag } 3 \\
r=.09, p=.67\end{array}$ & $\begin{array}{l}\text { Lag } 7 \\
\mathrm{r}=.47, p=.03^{*}\end{array}$ \\
\hline Spatial discontinuity Pre-T2 only & $\begin{array}{l}\operatorname{Lag} 2 \\
r=-22, p=.32\end{array}$ & $\begin{array}{l}\text { Lag } 3 \\
r=-.49, p=.02 *\end{array}$ & $\begin{array}{l}\text { Lag } 7 \\
r=-.15, p=.51\end{array}$ \\
\hline Spatial discontinuity Pre-T1 only & $\begin{array}{l}\operatorname{Lag} 2 \\
r=.43, p=.04 *\end{array}$ & $\begin{array}{l}\text { Lag } 3 \\
r=.56, p<.01^{*}\end{array}$ & $\begin{array}{l}\text { Lag } 7 \\
r=.07, p=.74\end{array}$ \\
\hline
\end{tabular}

temporal condition, these impairments were additive when the noise occurred in both stream locations.

The observed T1-T2 correlations enable interesting speculation, although we note that the $\mathrm{AB}$ literature is replete with opposing views on this matter. The absence of correlations in the $\mathrm{AB}$ interval in the temporal noise conditions is likely due to a ceiling effect. The positive correlations in the pre-T1 spatial noise condition are consistent with accounts (e.g., Chun \& Potter, 1995; Di Lollo, Kawahara, Ghorashi, \& Enns, 2005) in which processes affecting $\mathrm{T} 1$ exert an effect on $\mathrm{T} 2$. The negative correlation in the pre-T2 spatial noise condition is likely due to a combination of the effects of $\mathrm{T} 1$ on $\mathrm{T} 2$ as discussed and the spatial noise manipulation. The effect of noise, both temporal and spatial, is further evident in the tight coupling between $\mathrm{T} 1$ and $\mathrm{T} 2$ performance differences, as clearly can be seen in Fig. 2a,b. The implication is that noise facilitates or impedes target selection, respectively, over and above the direct effects of $\mathrm{T} 1$ on $\mathrm{T} 2$.

The hypothesis motivating the present experiment is that oscillatory brain patterns can play an important role in modulating cognitive processes such as those involved in the AB. A theory exemplifying the importance of oscillatory patterns is stochastic resonance, which suggests that a weak signal becomes more perceptible when accompanied by a small amount of noise. Empirical support for this notion has come from Kitajo et al. (2006), who showed a performance improvement in detecting a visual target adjacent to a spatial location where $20-\mathrm{Hz}$ gray-level noise was presented. Directly relevant to the hypothesis under consideration, Kawahara (2009) discovered improved performance in an $\mathrm{AB}$ task when $\mathrm{T} 2$ was accompanied by certain types of noise in the RSVP stream periphery. Although the noise arose from different sources, it was continuously present throughout the RSVP stream. Importantly, performance improvements were observed only when the noise arose from a category different from that of the target - for example, letter noise in a digit detection task. Noise arising from the same category-for example, digit noise accompanying a digit detection task-was found, instead, to reveal decreased performance.
As can be readily seen, the results of the present experiment conform to, yet extend, the findings from Kawahara's (2009) lab. Like Kawahara, we observed improved performance (i.e., less $\mathrm{AB}$ ) when the noise, temporal discontinuity, was from a dimension different from that of the spatial task required for letter target identification. Also consistent with Kawahara's findings, we saw a larger AB when the noise-in this case, spatial discontinuity - arose from a source similar to that required by the same target tasks.

Stochastic resonance theory specifies two conditions that must be met if the theory is to hold to its predictions. First, the stimulus to be enhanced must be subthreshold, a requirement met by the (T2) target occurring in the $\mathrm{AB}$ interval. Second, not all types of noise will augment performance-again, a condition met by our findings, as revealed by the opposite effects of temporal and spatial noise. Our results can thus be accommodated within the framework of stochastic resonance theory, since they meet both of these conditions. Their main novelty lies in extending the previous findings from Kawahara's (2009) lab, obtained with noise external to the RSVP stream, to variations in the noise associated with the stream itself. At the same time, we note one discrepancy with Kawahara's results, in which there were no effects of noise on the T1 task. In contrast, we found $\mathrm{T} 1$ rising and falling in concert with T2, for the most part, suggesting the possibility that some other mechanism may be operating with streamintrinsic noise.

Another account of the present results arises from electrophysiological evidence revealing that prestimulus alpha $(8-12 \mathrm{~Hz})$ oscillations predict behavioral performance. Long-distance (between brain regions) phase coupling in the alpha range has been correlated with highlevel processing during cognitive tasks in humans, such as manipulation or maintenance of information in working memory (Palva, Monto, Kulashekhar, \& Palva, 2010; Sauseng et al., 2005). In seeming contradiction, Hanslmayr et al. (2007) found the optimal conditions for target detection to arise under conditions of low prestimulus alpha phase coupling. This contradiction is resolved, 
however, if the conditions for external perception and those for internal processing are viewed as opposing states. In other words, it makes sense for the brain to cease inputting new information in order to process information previously received. Interruption masking, where a succeeding stimulus interrupts processing of a preceding stimulus, has the same effect but operates at a lower level in the visualprocessing hierarchy (cf. Brehaut, Enns, \& Di Lollo, 1999).

The 10-Hz RSVP rate used in the present and in virtually all $\mathrm{AB}$ experiments creates the antecedent conditions for variations in alpha oscillatory power to have the stated effect. The $\mathrm{AB}$, by the argument above, arises when alpha phase coupling is effected in the process of T1's being identified, in turn putting the brain in an internal state where it is no longer sensitive to external input and T2 identification suffers. Low phase coupling prior to $\mathrm{T} 1$ explains the accuracy associated with this target. To place the present results in this framework, we suggest that temporal noise interrupts phase coupling - in turn, facilitating T2, as well as T1, target perception. Spatial noise, on the other hand, and for reasons not yet clear, does not interrupt alpha phase coupling, leaving T2 in the state associated with poor detection. It is important to note that this account does not specify a relationship between the type of noise and the particular target task requirement, as underlies the application of stochastic resonance theory to the present findings.

The present results could also be viewed as related to the findings of Olivers and Nieuwenhuis $(2005,2006)$ that listening to a repetitive tune, thinking about one's holiday, performing an additional memory task, previewing affectively positive pictures, and focusing less deliberately on the $\mathrm{AB}$ task all result in dramatic reductions in the $\mathrm{AB}$. The paradoxical finding that various forms of distraction extrinsic to the stimulus stream can attenuate the $\mathrm{AB}$ (see also Arend, Johnston, \& Shapiro, 2006; Ho, Mason, \& Spence, 2007) is explained by assuming that the $A B$ arises from overinvestment of attentional resources into filtering for $\mathrm{T} 1$ and that distraction attenuates the overinvestment (cf. Olivers \& Meeter, 2008) and the subsequent blink. Although this hypothesis was not formulated to account for intrinsic forms of distraction - that is, noise arising from variations in the RSVP stream - the present finding from the temporal noise discontinuity condition is consistent with the account above. On the other hand, noise arising from the spatial discontinuity condition is inconsistent with the overinvestment account.

To summarize, the present data provide an important impetus for those seeking neurally inspired accounts of the $\mathrm{AB}$ based on oscillatory patterns, as in the mechanisms of stochastic resonance and alpha phase coupling. To our knowledge, no current account of the $\mathrm{AB}$ can accommodate the present results that intrinsic discontinuities of the RSVP stream from temporal and spatial sources can produce increases and decreases in $\mathrm{AB}$ performance, respectively.

Acknowledgments This research was funded by Wellcome Trust Project Grant GR071944 to K. Shapiro and was supported by a Discovery Grant (NSERC) to J. Enns.

\section{References}

Arend, I., Johnston, S., \& Shapiro, K. L. (2006). Task-irrelevant visual motion and flicker attenuates the attentional blink. Psychonomic Bulletin \& Review, 13, 600-607.

Brehaut, J. C., Enns, J. T., \& Di Lollo, V. (1999). Visual masking plays two roles in the attentional blink. Perception \& Psychophysics, 61, 1436-1448.

Busch, N. A., Dubois, J., \& VanRullen, R. (2009). The phase of ongoing EEG oscillations predicts visual perception. Journal of Neuroscience, 29(24), 7869-7876.

Buzsáki, G. (2004). Large-scale recordings of neuronal ensembles. Nature Neuroscience, 7(5), 446-51.

Chun, M. M., \& Potter, M. C. (1995). A two-stage model for multiple target detection in rapid serial visual presentation. Journal of Experimental Psychology: Human Perception and Performance, 21, 109-127.

Di Lollo, V., Kawahara, J., Ghorashi, S. M. S., \& Enns, J. T. (2005). The attentional blink: Resource depletion or temporary loss of control. Psychological Research, 69, 191-200.

Dux, P. E., \& Marois, R. (2009). The attentional blink: A review of data and theory. Attention, Perception, \& Psychophysics, 71, 1683-1700.

Gammaitoni, L., Hanggi, P., Jung, P., \& Marchesoni, F. (1998). Stochastic resonance. Review of Modern Physics, 70, 223-287.

Hanslmayr, S., Asian, A., Staudigl, T., Klimesch, W., Herrmann, C. S., \& Bäuml, K.-H. (2007). Prestimulus oscillations predict visual perception performance between and within subjects. Neuroimage, 37, 1465-1473.

Ho, C., Mason, O., \& Spence, C. (2007). An investigation into the temporal dimension of the Mozart effect: Evidence from the attentional blink task. Acta Psychologica, 125, 117-128.

Kawahara, J. (2009). When do additional distractors reduce the attentional blink? Journal of Experimental Psychology: Human Perception and Performance, 35, 1043-1061.

Kitajo, K., Yamanaka, K., Ward, L. M., \& Yamamoto, Y. (2006). Stochastic resonance in attention control. Europhysics Letters, 76, 1029-1035.

Olivers, C. N. L., \& Meeter, M. (2008). A boost and bounce theory of temporal attention. Psychological Review, 115, 836-863.

Olivers, C. N. L., \& Nieuwenhuis, S. (2005). The beneficial effect of concurrent task-irrelevant mental activity on temporal attention. Psychological Science, 16, 265-269.

Olivers, C. N. L., \& Nieuwenhuis, S. (2006). The beneficial effects of additional task load, positive affect, and instruction on the attentional blink. Journal of Experimental Psychology: Human Perception and Performance, 32, 364-379.

Palva, J. M., Monto, S., Kulashekhar, S., \& Palva, S. (2010). Neuronal synchrony reveals working memory networks and predicts individual memory capacity. Proceedings of the National Academy of Sciences, 107, 7580-7585.

Raymond, J. E., Shapiro, K. L., \& Arnell, K. M. (1992). Temporary suppression of visual processing in an RSVP task: An attentional blink? Journal of Experimental Psychology: Human Perception and Performance, 18, 849-860.

Sauseng, P., Klimesch, W., Doppelmayr, M., Pecherstorfer, T., Freunberger, R., \& Hanslmayr, S. (2005). EEG alpha 
synchronization and functional coupling during top- down processing in a working memory task. Human Brain Mapping, 26, 148-155.

Schultz, D. W., \& Eriksen, C. W. (1978). Stimulus size and acuity in information processing. Bulletin of the Psychonomic Society, 12, 397-399.
Seiffert, A. E., \& Di Lollo, V. (1997). Low-level masking in the attentional blink. Journal of Experimental Psychology: Human Perception and Performance, 23, 1061-1073.

Wiesenfeld, K., \& Moss, F. (1995). Stochastic resonance and the benefits of noise: From ice ages to crayfish and SQUIDs. Nature, $373,33-36$. 